\title{
Orbital mold brachytherapy for recurrent orbital mesenchymal chondrosarcoma: a case report
}

\author{
Eugene Yap, MD, Stellar Cabrera, MSc in Applied Physics, Maureen Bojador, MSc in Medical Physics, Teresa Sy Ortin, MD, \\ Warren Bacorro, MD \\ University of Santo Tomas Hospital, Benavides Cancer Institute, Manila, Philippines; University of Santo Tomas Faculty of Medicine and \\ Surgery, Manila, Philippines
}

\begin{abstract}
Purpose: The aim of this case report is to present a case of orbital mesenchymal chondrosarcoma sarcoma with multiple recurrences, and to report technical details of a contemporary approach for orbital brachytherapy that can be used in low-resource settings.

Material and methods: A 46-year-old female diagnosed with recurrent orbital mesenchymal chondrosarcoma of the left orbit presented with her third local recurrence. The patient proceeded with conservative surgery with planned adjuvant high-dose-rate brachytherapy 2 weeks post-surgery. Brachytherapy mold applicator was fabricated using thermoplastic mask, ProGuide catheter needles, catheter fixation buttons, and a strip of gauze. Ideal catheter placement was done with CT simulation planning.

Results: High-risk clinical target volume (HR-CTV) corresponded to gross tumor residual, and intermediate-risk clinical target volume (IR-CTV) corresponded to the whole orbit. Flexitron iridium-192 high-dose-rate (HDR) brachytherapy plan was generated using Oncentra brachytherapy planning system. The treatment plan had HR-CTV total dose of 55.6 Gy and equivalent dose in 2 Gray fractions $\left(\mathrm{EQD}_{2}\right)$ of $96 \mathrm{~Gy}$. IR-CTV total dose was $45.5 \mathrm{~Gy}$ and EQD $\mathrm{EQ}_{2}$ of Gy. The plan was evaluated using dose-volume histogram and dosimetric parameters, which showed adequate irradiation of tumor volume and at-risk areas. The patient underwent orbital brachytherapy without any adverse events, except for mild skin erythema. One-year post-treatment showed no local recurrence and no soft tissue necrosis or swelling.

Conclusions: In the previously irradiated orbital sarcoma patient, adjuvant brachytherapy is an effective and safe modality for the delivery of sufficient radiation dose to the target, as presented in the current case report. The materials used in the brachytherapy applicator are easily available in majority of radiation therapy centers and can be manufactured even in low-resource settings.
\end{abstract}

Key words: orbit, brachytherapy, high-dose-rate, sarcoma, case report.

\section{Purpose}

Orbital tumors have an incidence rate of 1.59 per million person/year, with majority consisting of carcinomas, melanomas, sarcomas, and lymphomas [1]. The management of orbital tumors would depend on the type of tumor and its extent of invasion. Surgical interventions include exenteration, enucleation, or excision with wide margins. However, patients with advanced diseases usually preclude total resection of all gross tumors, which is the most common indication for post-operative radiation therapy, as shown in multiple studies reporting adjuvant radiotherapy reducing local recurrence rates [2]. Radiation therapy can be administered either with external beam radiotherapy or brachytherapy, each with its own advantages and disadvantages. Currently, published reports on orbital brachytherapy show various types of ap- plicators, ranging from intracavitary molds applicators to interstitial brachytherapy [3-8]. The most optimal applicator would be a patient-specific 3D-printed applicator, as this would conform with the anatomy of the patient [8]. However, due to financial reasons, it is not very practical to use, especially in low-resource centers. In addition, interstitial brachytherapy would entail expertise not available to most centers. Here, we reported technical details of a contemporary approach for orbital brachytherapy used in our institution, which can be applied in low-resource settings without compromising dose coverage.

\section{Case details}

A 46-year-old female with recurrent orbital mesenchymal chondrosarcoma of the left orbit presented with her third local recurrence. Eight years prior, the patient
Address for correspondence: Eugene Richard T. Yap, Department of Radiation Oncology, Benavides Cancer Institute, University of Santo Tomas Hospital, España, Manila, Philippines 1015, e-mail: eugene.tan.yap@gmail.com
Received: 26.07.2021

Accepted: 19.09 .2021

Published: 30.12 .2021 
initially presented with exophthalmos, diplopia, and proptosis of the left eye. Imaging showed a $3 \mathrm{~cm}$ extraconal mass, abutting the laterally displaced left medial rectus muscle, and excision revealed a histopathology of extraskeletal mesenchymal chondrosarcoma. Imaging showed a residual mass; therefore, the patient was started on 6 cycles of chemotherapy using Ewing sarcoma's protocol, resulting in complete regression of the residual tumor. Four years after the initial treatment, there was a recurrence of the mass, and the patient underwent sub-total resection using adjuvant chemotherapy with external beam radiation therapy to $55.8 \mathrm{~Gy}$ to the left orbit, with complete clinical response. Three years after that treatment, there was another recurrence, and orbital exenteration of the left orbit was performed. There was a positive deep margin on final histopathology; therefore, during a multidisciplinary meeting with the patient, it was decided to reserve chemotherapy for metastatic progression and to proceed with adjuvant re-irradiation for local control. The risks and benefits of adjuvant treatment were explained to the patient, but she was reluctant to proceed with it due to the risk of osteonecrosis. Less than a year after the surgery, the patient experienced a third local recurrence.
Imaging showed a soft tissue mass with a maximum diameter of $3 \mathrm{~cm}$ within the left orbit, coursing along the lateral aspect involving the orbital rim and orbital floor into the superior orbital fissure, optic canal, and inferior orbital fissure. Since the patient has already received multiple prior treatments, consisting of excisions, orbital exenteration, multiple lines of chemotherapy, and external beam radiotherapy, a multi-disciplinary meeting was conducted, during which it was decided to proceed with repeated surgery with maximal debulking and planned adjuvant high-dose-rate (HDR) brachytherapy 2 weeks post-surgery, with chemotherapy reserved for metastatic progression.

\section{Material and methods}

\section{Applicator design}

One week prior to the surgery, the orbital mold brachytherapy applicator was manufactured. With the patient in supine position, with the neck positioned neutrally, the applicator was shaped using a thermoplastic mask molded to follow the contours of orbit, and with a portion molded to follow the bony prominences of uninvolved face, which served as docking portion for re-

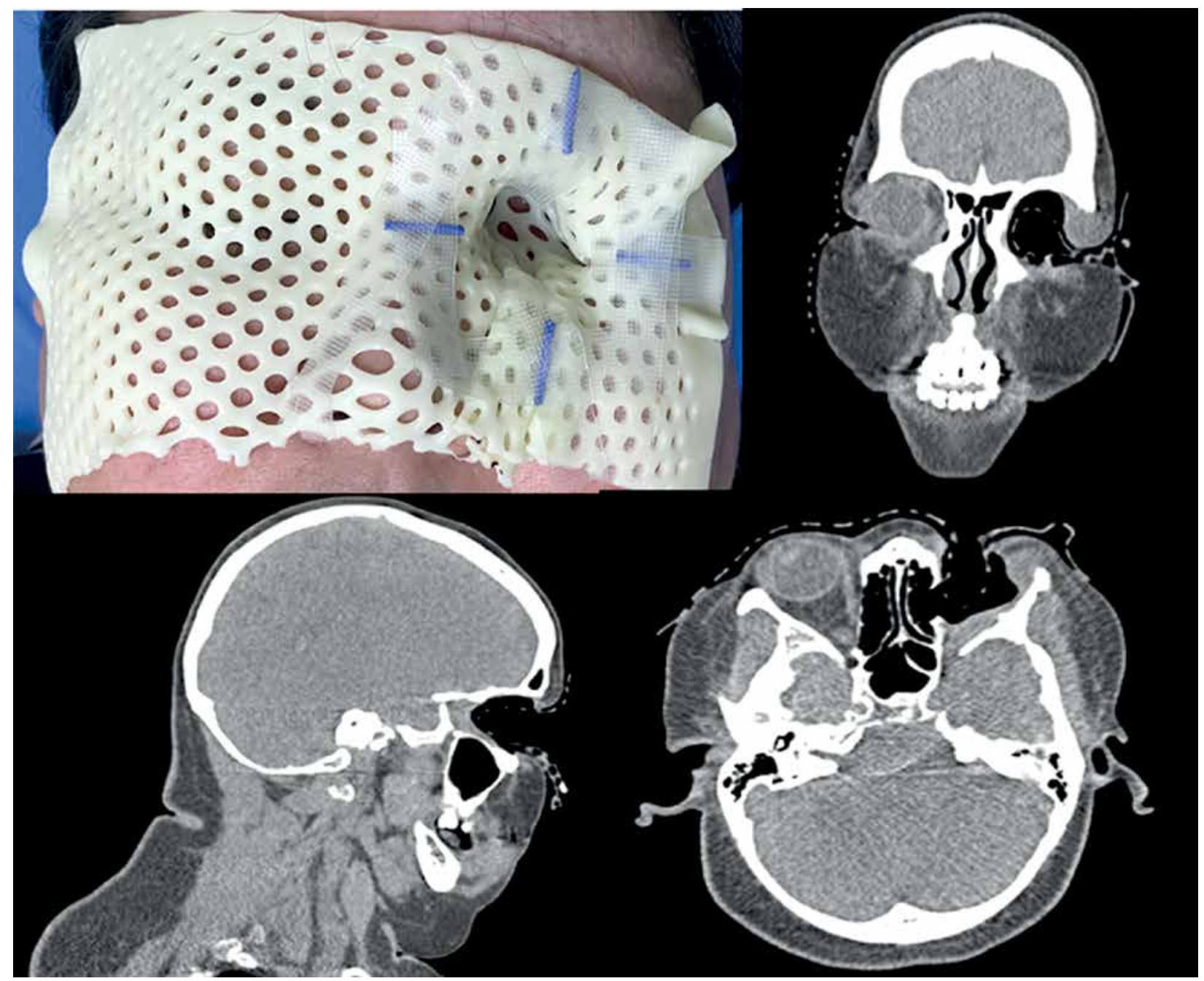

Fig. 1. Application design CT simulation 
producibility of mold placement. The orbital portion was molded with a tapering or conoid shape to ensure unobstructed removal. By producing the mold pre-operatively, topography of the tumor was imprinted on the orbital portion of the mask and later-guided catheter configuration. The patient underwent a $1 \mathrm{~mm}$ slice thickness CT simulation scan with the mold in place to visualize and evaluate ideal catheter placement together with the catheter numbers, positioning, depth, and length (Figure 1). Also, the estimated extent of surgical resection and projected clinical target volumes (pCTV) were delineated on CT simulation scan. ProGuide catheter needles were inserted through perforations in the thermoplastic mask, assembled around the orbital mold and secured loosely at the apex using fixator buttons. Tumor-subtracted CT simulation images were used as a guide for evaluating feasibility of physical configuration. ProGuide catheter needles were tentatively positioned trough an incision made on the mask that corresponded to the bulk of tumor and soft tissue to be resected. Each physical configuration was evaluated based on projected distance from pCTV. Several re-repetitions were done to achieve the optimal dosimetry and feasible configuration. The optimal configuration was found with the use of 10 flexible ProGuide needles placed 5-8 mm apart from each other, converging on the apex of the mold to cover the whole orbit. They were inserted though the thermoplastic mask perforations with similar depths, except for needle No. 3, which was positioned deeper to adequately cover the optic canal. Fixator buttons were glued to the apex to maintain spacing and allow further advancement of the needles as needed based on the actual post-operative bed. A thin layer of gauze was applied to further secure the needles along the trunk of the mold (Figure 2).

\section{Pre-planning}

Two weeks post-operatively, the orbital mold applicator was again fitted to assure reproducibility of daily positioning. Lidocaine spray was applied to relieve postop dysesthesia/allodynia. Despite removal of soft tissue surrounding the orbit, the applicator was still fixed and

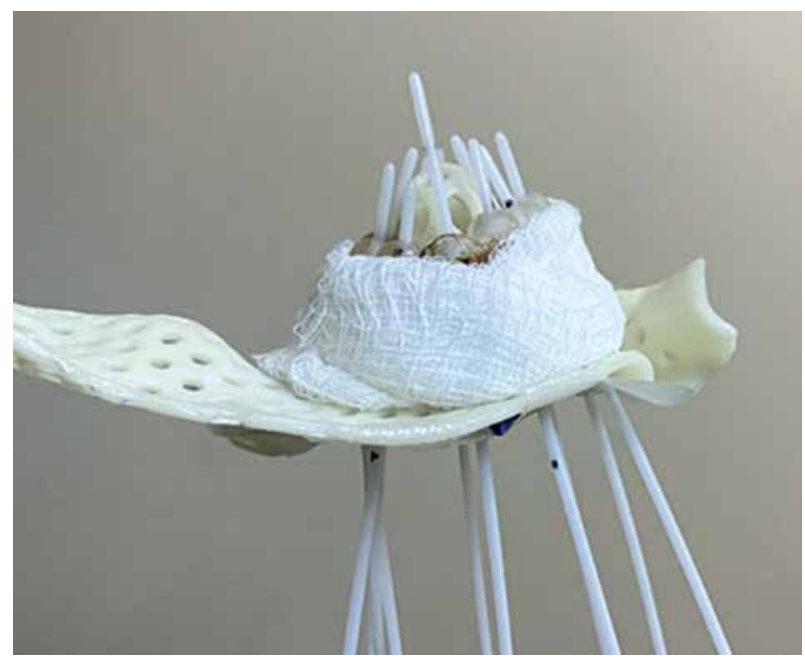

Fig. 2. Orbital mold brachytherapy applicator reproducible due to multiple points of contact with facial bony prominences (glabella and zygoma). Final configuration of ProGuide needles was then adjusted according to post-operative orbital anatomy. Tape was used to secure the applicator. CT markers were inserted through the needles for visualization during CT simulation of $1 \mathrm{~mm}$ slice thickness. Adjustments were made to the needles' depth as needed until CT simulation showed satisfactory coverage of the whole orbit. CT markers were reconstructed in the planning system and converted as a radioactive source path (Figure 3). Measurement of each catheter was performed for reproducibility.

\section{Results}

\section{Planning and dosimetry}

High-risk clinical target volume (HR-CTV) corresponded to gross tumor prior to resection, and was contoured together with the surgeon to identify the sites of higher risk of recurrence, which included areas abutting the gross tumor or areas wherein the bone was shaved off during surgery. This further corresponded with the apex, inferior, and right and left lateral walls of the orbit. Intermediate-risk clinical target volume (IR-CTV) was contoured and corresponded to the whole orbit. The contralateral eye and lens were also contoured for monitoring. Flexitron iridium-192 HDR brachytherapy plan was generated using Oncentra brachytherapy planning system v. 4.5.2 (Oncentra Brachy, Elekta AB, Sweden). The plan was generated using ten channels, single unidirectional stepwise moving source, and remote afterloading with flexible catheters, with a dwell step size of $0.2 \mathrm{~cm}$. Treatment surface was curved, and the implant geometry was planar with a conoid shape. Graphical dose optimization technique was used.

The planned dosimetry was HR-CTV $\mathrm{D}_{90} \geq 3.5-4$ Gy and IR-CTV $D_{95} \geq 3-3.5$ Gy in 14 twice-daily fractions 6-hours apart. This was actually delivered in 7 twice-daily fractions per week, with weekend break, and thus an overall treatment time was 10 days. Assuming no tumor repair in the 6-hours interfraction interval for twice-daily

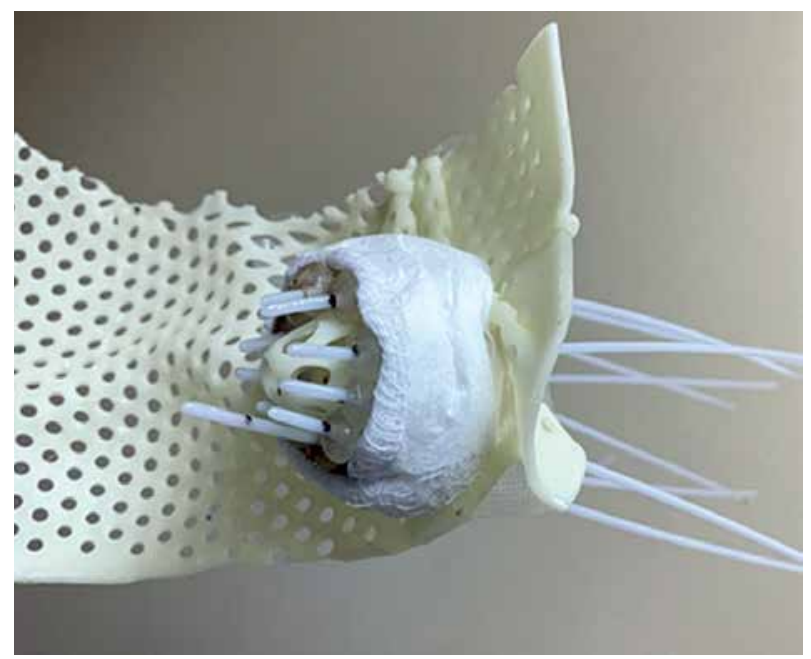



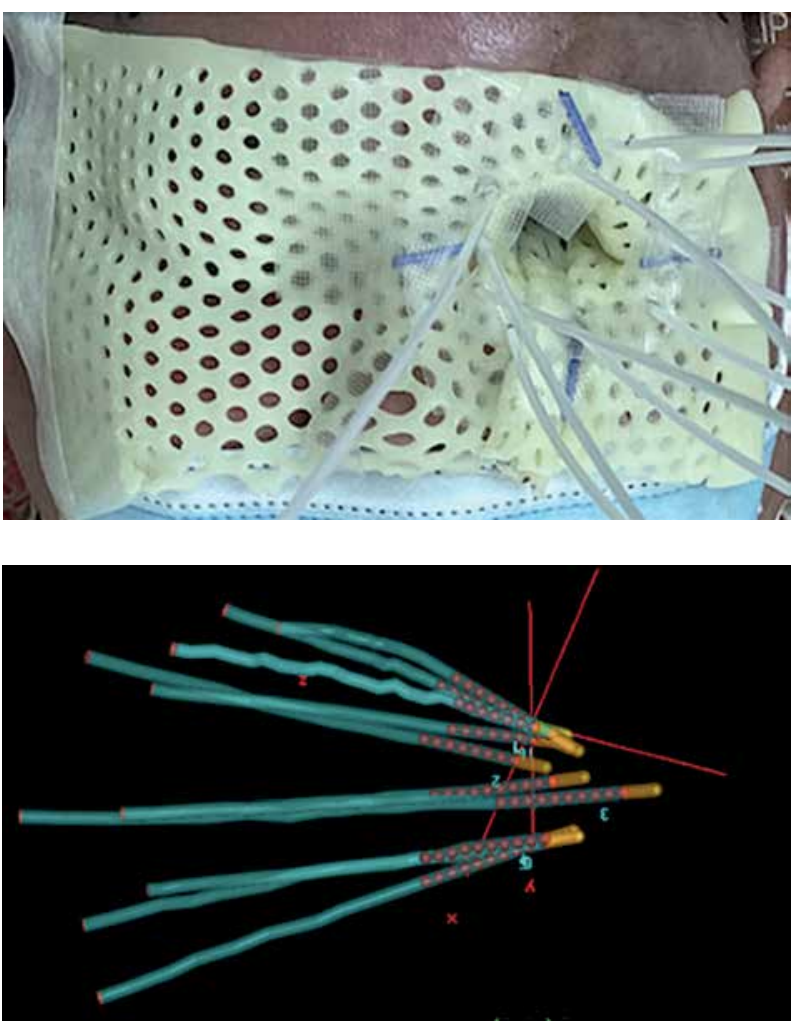

fractions, the dose would be HR-CTV $\mathrm{D}_{90} \geq 7-8$ Gy/day, and IR-CTV $D_{95} \geq 6-7$ Gy/day [9]. With sarcomas having an $\alpha / \beta$ ratio of 5.4 [10], HR-CTV total dose would result in 49-56 Gy with EQD 2 of 78.8-97.1 Gy. For IR-CTV, the total dose would be $42-49 \mathrm{~Gy}$, with $\mathrm{EQD}_{2}$ of $62.3-78.8 \mathrm{~Gy}$.

The accepted plan was estimated, including HR-CTV $\mathrm{D}_{90}$ of $3.97 \mathrm{~Gy} /$ fraction, with a total dose of $55.6 \mathrm{~Gy}$ and $\mathrm{EQD}_{2}$ of 96 Gy. IR-CTV $\mathrm{D}_{95}$ was $3.25 \mathrm{~Gy} /$ fraction, with a total dose of $45.5 \mathrm{~Gy}$ and $\mathrm{EQD}_{2}$ of $70 \mathrm{~Gy}$. For normal tissues, an $\alpha / \beta$ ratio of 10 was applied for acute effects, resulting $\mathrm{EQD}_{2}$ of $60.8 \mathrm{~Gy}$ and $80.5 \mathrm{~Gy}$ for IR-CTV and HR-CTV, respectively. For late effects, $\alpha / \beta$ ratio of 3 was used, which resulted to EQD $\mathrm{E}_{2}$ of $82.2 \mathrm{~Gy}$ for IR-CTV and 115.0 Gy for HR-CTV (Figure 4, Table 1). Doses received by the contralateral orbit and lens were also monitored with the lens $\mathrm{D}_{0.1 \mathrm{cc}}$ receiving a dose of $0.36 \mathrm{~Gy} /$ fraction, with total dose of $5 \mathrm{~Gy}$ and $\mathrm{EQD}_{2}$ of $3.7 \mathrm{~Gy}$. The contralateral orbit received $0.5 \mathrm{~Gy} /$ fraction, with total dose of $7 \mathrm{~Gy}$ and $\mathrm{EQD}_{2}$ of $5.5 \mathrm{~Gy}$. The plan was evaluated to ensure targets receiving appropriate doses and sufficient coverage, while organs at risk not getting a significant dose. The treatment planning and refinement took 2 days until the plan was accepted, and the patient started treatment immediately the next day.

\section{Treatment delivery and outcomes}

Prior to treatment, lidocaine spray was applied to the orbital region due to post-operative hyperesthesia. The patient was in supine position, and the applicator appropriately located and secured, making sure catheter No. 3 abuts the optic canal and the four points of contact were positioned. Measurement of each catheter was ver-

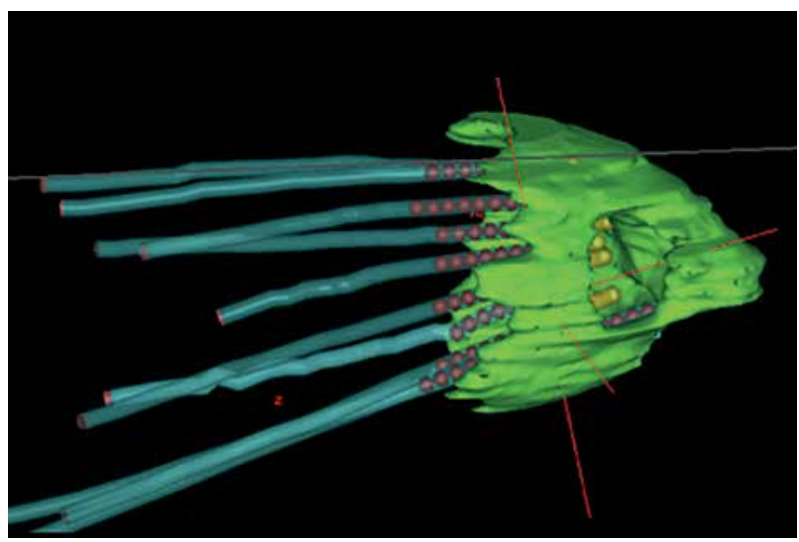

Fig. 3. Applicator 3D reconstruction. Cyan - reconstructed ProGuide catheters, green - IR-CTV, red dots - dwell points

ified prior to each treatment. CT markers were used for position localization and orthogonal radiographs were taken (Figure 5). Placement was verified by comparing the orthogonal radiograph to the digitally reconstructed radiography (DRR). CT markers were removed, and the catheters were connected to transfer tubes. Then, the treatment was delivered.

The patient underwent orbital brachytherapy in 14 sessions. The treatment was without any adverse events, except for mild skin erythema (according to CTCAE v. 5.0, grade 1 radiation dermatitis) observed on the $10^{\text {th }}$ session of treatment, which persisted until 2-week follow-up. The regimen was well-tolerated, with healthy tissue granulation observed during follow-up, two weeks after the procedure. One-year post-treatment showed no local recurrence, and no soft tissue necrosis or swelling.

\section{Discussion}

Radiation therapy for orbital tumors can be performed with either external beam radiotherapy or brachytherapy. External beam radiotherapy can reach deeper structures and is not limited by the constraints of accessibility to treatment site. However, such treatment has been associated with toxicities of normal tissues surrounding treated area, including retinopathies, cataracts, eyelash loss, eyebrow loss, dry socket, socket retraction, and cranio-facial deformities [11, 12]. In addition, re-irradiation with external beam radiotherapy is associated with greater normal tissue toxicity. On the other hand, due to steep dose fall-off, brachytherapy can deliver adequate therapeutic dose to the target and reduced dose to surrounding 

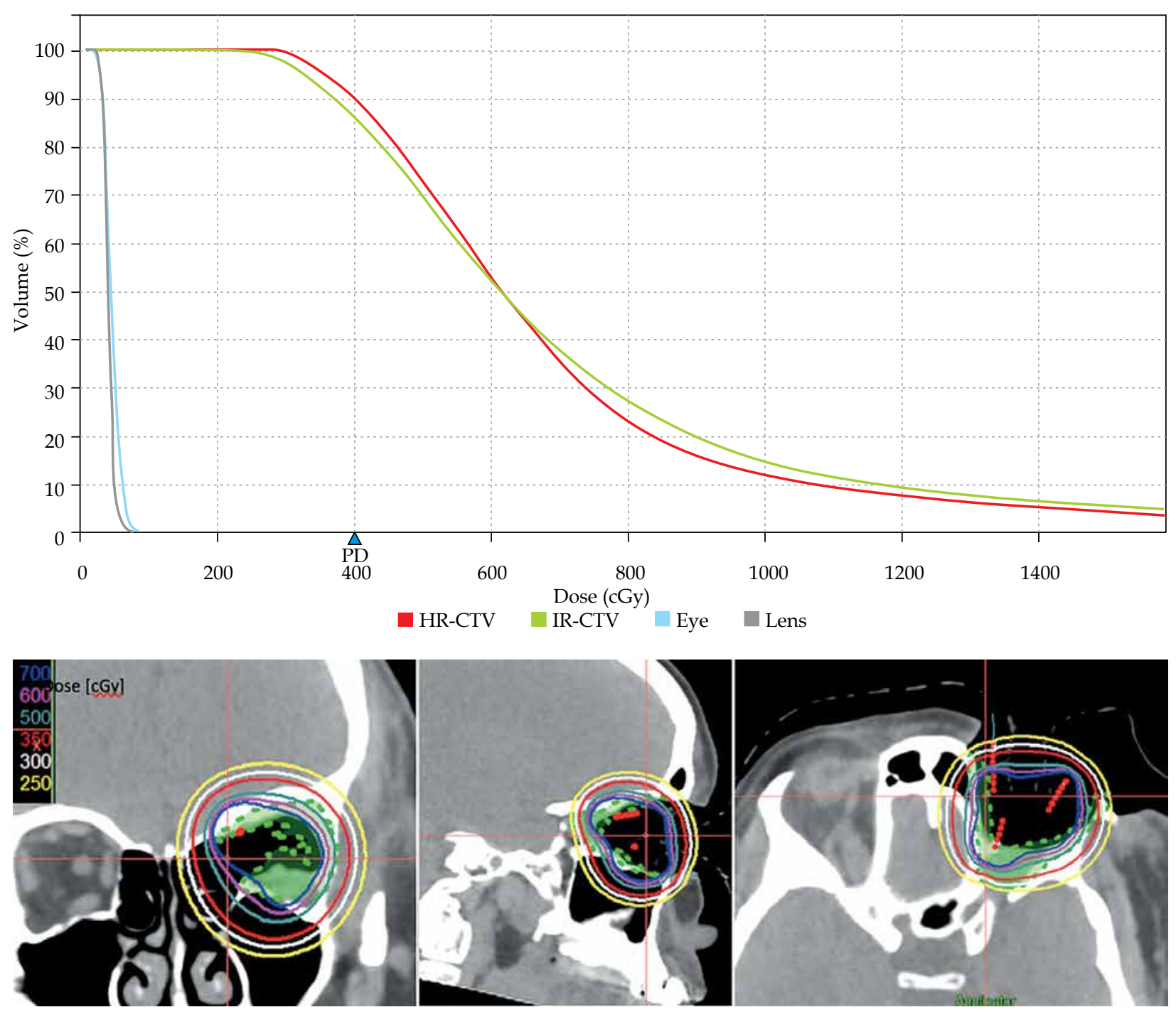

Fig. 4. Dose-volume histogram. Red line - HR-CTV, green line - IR-CTV, blue line - contralateral eye, gray line - contralateral lens

Table 1. Treatment dosimetry

\begin{tabular}{lcccc}
$\begin{array}{l}14 \text { fractions, } \\
\text { BID treatment, } \\
\text { high-dose-rate }\end{array}$ & IR-CTV $D_{95}$ & HR-CTV $D_{90}$ & Eye $D_{2 c c}$ & Lens $D_{0.1 c c}$ \\
\hline Target & Whole orbit & $\begin{array}{l}\text { Apex, inferior, right } \\
\text { and left lateral walls }\end{array}$ & Contralateral eye & Contralateral lens \\
\hline Dose received/fraction (Gy) & 3.25 & 3.97 & 0.5 & 0.36 \\
\hline Total dose $(G y)$ & 45.5 & 55.6 & 7.0 & - \\
\hline$E Q D_{2}(\alpha / \beta=5.4)(G y)$ & 70.3 & 96.1 & - & - \\
\hline $\begin{array}{l}\text { Acute effects of } \mathrm{EQD}_{2} \\
(\alpha / \beta=10)(G y)\end{array}$ & 60.8 & 80.5 & & 3.0 \\
\hline Late effects of $\mathrm{EQD}_{2}(\alpha / \beta=3)(G y)$ & 82.2 & 115.0 & 5.5 & 3.7
\end{tabular}

normal tissues. Therefore, it reduces toxicities and allow for radiation treatment of previously irradiated sites [13, 14]. Brachytherapy also leads to decreased late toxicity compared to external beam radiation therapy, since surrounding normal tissues do not receive a significant dose of radiation, and because the radiation source emerges from the orbital cavity without an entry and exit of dose through normal tissue. However, this present a drawback of not being able to penetrate deeper tissues, and it is technically more difficult to administer. Moreover, it is 
further complicated by irregular tissue contours, lack of specific site applicators, and deformed anatomy.

With chondrosarcomas, the National Comprehensive Cancer Network (NCCN) recommends an adjuvant dose of $70 \mathrm{~Gy}$ for R1 resection and 72-78 Gy for R2 resection [15]. With HR-CTV corresponding to gross residual and IR-CTV for microscopic tumor, our plan adequately met the recommendation. IR-CTV EQD ${ }_{2}$ for the case presented was $70.3 \mathrm{~Gy}$, and HR-CTV EQD 2 was $96 \mathrm{~Gy}$. The main objective for the treatment was to deliver adequate doses to both HR-CTV and IR-CTV to maximally decrease local risk of recurrence, given that salvage local treatment modalities would be limited in the future. To deliver adequate dose to IR-CTV, a higher-than-prescribed dose to HR-CTV had to be accepted. The escalated dose would not be a challenge in terms of toxicity due to delivery of focal doses and effects of an inverse-square law, with exponential decline in dose in relation to the distance. The contralateral orbit and lens received a negligible dose, with adverse effects not expected. Due to high-dose given, the most challenging adverse event would be osteoradionecrosis, which is a rare complication that may present years after the radiation treatment due to obliterative endarteritis and subsequent aseptic avascular necrosis. Close follow-up is essential for early identification and management, which includes analgesia, antibiotics, debridement, and in more severe cases, grafting [16].

Sarcomas have an $\alpha / \beta$ ratio of 5.4 , which is relatively lower than carcinomas [17]. Lower $\alpha / \beta$ ratio enhances the improvement of therapeutic index with hypofractionation without augmenting toxicity risk, since acute effects would be less, while maintaining late side effects. Acute effects have a relatively high $\alpha / \beta$ ratio, hence, when looking at the equivalent dose in 2 Gy fractions, the dose for acute effects is relatively lower than that of target dose. Furthermore, giving the doses twice-daily with at least 6-hours apart, allows for repairing of normal tissue, but not of the tumor, therefore, improving therapeutic index.

The orbital mold brachytherapy technique reported in this article provided a detailed approach on the manufacture of the applicator, and showed excellent coverage of the orbit without the need for interstitial brachytherapy or other more expensive applicators. The materials used are easily accessible and do not require extensive technical skills in fabrication. The thermoplastic mask is universally used in radiation oncology departments for immobilization. The perforations of the thermoplastic mask are convenient for securing and stabilizing the ProGuide needles. Additionally, catheter fixator buttons were used to allow adjustment to the desired depth. With the addition of a thin gauze for additional stability, it affords a softer and smoother base for better comfort during application. Because of initial adjustability, certain areas could be covered by positioning of some catheters deeper, similarly to covering of the apex.

With careful selection of patients and optimal planning, brachytherapy of the orbit seems to be effective if the treated area is adequately covered, and limited dos-

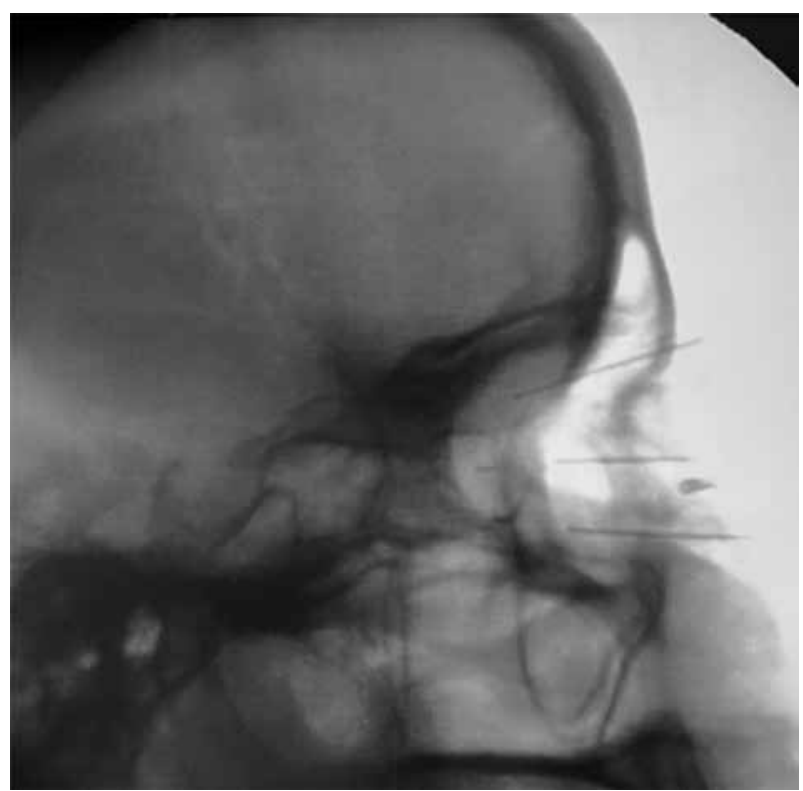

Fig. 5. Localizing the position with orthogonal radiograph

es received by other organs are maintained. A long-term follow-up is recommended since radiation therapy can produce late adverse effects, especially for re-irradiated patients.

\section{Conclusions}

In a previously irradiated orbital sarcoma patient, adjuvant brachytherapy is an effective and safe modality for the delivery of sufficient radiation dose to the target, as seen in the treatment and follow-up of the patient in the presented case report. The materials used in brachytherapy application are easily available in majority of radiation therapy centers, which can be manufactured even in a low-resource setting.

\section{Ethical considerations}

This study was reviewed by research ethics committee of the author's institution. It was conducted in accordance with the Declaration of Helsinki, WHO Operational Guidelines in Biomedical Studies 2011, International Conference on Harmonization on Good Clinical Practice (ICH-GCP), Council for International Organizations for Medical Sciences 2016, Good Research Practice (GRP), and the National Ethical Guidelines for Health and Health-Related Research of 2017 as well as the Philippine Data Privacy Act of 2012 and its' implementing rules and regulations (IRR) of 2016.

\section{Privacy and confidentiality}

Patient data were kept private and confidential in accordance with the Data Privacy Act of 2012 and its' implementing rules and regulations of 2016. Data and images used were anonymized and re-identified. Only necessary personal data was collected for the purpose of this study. Only the investigators had access to the original patient's data and images. Soft copies of data were stored in a pass- 
word-protected file in an external storage drive dedicated to this case report. All data were stored for safekeeping for five years. If deemed no longer needed, data will be permanently deleted from the drive.

\section{Informed consent process}

The patient was invited to participate in this study by allowing the investigators to use her information in this case report. An informed consent form was provided to the patient, with sufficient time to be read, understood, and clarified. The primary investigator administered the informed consent process and answered necessary questions. The patient was given a copy of the consent form, which was signed by the patient, the primary investigator, and an impartial witness. The principle of autonomy and respect was ensured at all time throughout this study.

\section{Disclosure}

The authors report no conflict of interest.

\section{References}

1. Hassan W, Bakry M, Hassan H et al. Incidence of orbital, conjunctival and lacrimal gland malignant tumors in USA from surveillance, epidemiology and end results, 1973-2009. Int J Ophthalmol 2016; 9: 1808-1813.

2. Mahvi D, Liu R, Grinstaff M et al. Local cancer recurrence: the realities, challenges, and opportunities for new therapies. CA Cancer J Clin 2018; 68: 488-505.

3. Tyl J, Blank L, Koornneef L et al. Brachytherapy in orbital tumors. Ophthalmology 1997; 104: 1475-1479.

4. Kim T, Gerbi B, Deibel F et al. An afterloading brachytherapy device utilizing thermoplastic material. Radiother Oncol 1989; 15: 341-344.

5. Abramson D, Fass D, McCormick B et al. Implant brachytherapy: A novel treatment for recurrent orbital rhabdomyosarcoma. J AAPOS 1997; 1: 154-157.

6. Laskar S, Pilar A, Khanna N et al. Interstitial brachytherapy for orbital soft tissue sarcoma: An innovative technique. J Contemp Brachytherapy 2017; 9: 466-471.

7. Finger P, Tena L, Semenova E et al. Extrascleral extension of choroidal melanoma: Post-enucleation high-dose-rate interstitial brachytherapy of the orbit. Brachytherapy 2014; 13 : 275-280.

8. Subashi E, Jacobs C, Hood R et al. A design process for a 3D printed patient-specific applicator for HDR brachytherapy of the orbit. 3D Print Med 2020; 6: 1-5.

9. Joiner M, Kogel A. Basic clinical radiobiology. Chapter 9. $4^{\text {th }}$ ed. CRC Press, Abingdon, Oxon 2009.

10. Soyfer V, Corn B, Kollender Y et al. Radiation therapy for palliation of sarcoma metastases: A unique and uniform hypofractionation experience. Sarcoma 2010; 2010: 927972.

11. Finger PT. Radiation therapy for orbital tumors: concepts, current use, and ophthalmic radiation side effects. Surv Ophthalmol 2009; 54: 545-568.

12. Nasser Q, Gombos D, Williams M et al. Management of radiation-induced severe anophthalmic socket contracture in patients with uveal melanoma. Ophthal Plast Reconstr Surg 2012; 28: 208-212.

13. Itami J. Modern development of high-dose-rate brachytherapy. Jpn J Clin Oncol 2021; 50: 490-501.

14. Marwaha G, Macklis R, Singh A et al. Brachytherapy. Dev Ophthalmol 2013; 52: 29-35.
15. National Comprehensive Cancer Network. Bone Cancer (Version 1.2022). http://www.nccn.org/professionals/physician_gls/pdf/bone.pdf

16. Yuhan B, Nguyen B, Svider P et al. Osteoradionecrosis of the temporal bone: An evidence-based approach. Otol Neurotol 2018; 39: 1172-1183.

17. Turesson I, Thames HD. Repair capacity and kinetics of human skin during fractionated radiotherapy: Erythema, desquamation, and telangiectasia after 3 and 5 year's follow-up. Radiother Oncol 1989; 15: 169-188. 